\title{
NOMENCLATURE OF SYZYGIUM GRACILE (MYRTACEAE)
}

\author{
J.F. VELDKAMP \\ Nationaal Herbarium Nederland, Universiteit Leiden branch, \\ P.O. Box 9514, 2300 RA Leiden, The Netherlands; \\ e-mail: veldkamp@nhn.leidenuniv.nl
}

\section{SUMMARY}

Syzygium glabratum is the correct name for Syzygium gracile.

Key words: Syzygium glabratum, Syzygium gracile, Syzygium ramosissimum, Myrtaceae, Malesia.

Ms.Amshoff (1945) coined the combination Syzygium gracile (Korth.) Amshoff, based on Jambosa gracilis Korth. (1847). She cited an apparently earlier place where the combination would have been made: the 'Noodflora voor Java' (1944). However, all new taxa and combinations found there must be regarded as invalid, as the Noodflora was prepared to safeguard Backer's manuscripts in a number of places. Hence the publication in 1945 was necessary to validate some new names and taxa.

However, Amshoff also cited Myrtus glabrata Blume (1826-1827: 1083, '1088'), which is a later homonym of $M$. glabrata $\mathrm{Sw}$. (1788). She was apparently not aware that Blume's taxon was given a valid name, Jambosa glabrata, by De Candolle (1828), i.e. older than Korthals' name. Syzygium gracile (Korth.) Amshoff is superfluous: 'glabratum' should have been used, as there seems to be no Syzygium glabratum then and now (IPNI, May 21, 2003).

The synonymy of the taxon is:

Syzygium glabratum [Blume] (DC.) Veldk., comb. nov.

Myrtus glabrata Blume, Bijdr. 17 (1826-1827) 1083, non Sw. (1788). - Jambosa glabrata DC., (1828) 287. - Clavimyrtus glabrata Blume (1850) 114. - Eugenia blumeana Kuntze (1891) 239, nom. nov., non E. glabrata DC. (1828). - Lectotype of Clavimyrtus Blume, designated by Merr. \& Perry [(1939) 141 ('Myrtus glabrata DC.')]. - Type: Blume 561 (holo L, sh. 908.1462999), designated here [Henderson, (1949) 249, said to have seen the type, but no sheet in L is marked by him].

Jambosa gracilis Korth. (1847) 202. - Eugenia clavimyrtus Koord. \& Valeton (1900) 110, nom. superfl. - Syzygium gracile Amshoff in Backer [(1944) 22, nom. inval.], (1945) 500, nom. superfl.; Merr. (1950) 392; Backer \& Bakh.f. (1964) 343; I.M. Turner (1996) 15; (1997, '1995') 376. - Type: Korthals s.n., 'in cultis agri bogoriensis' [not found, but presence in L cited by Merrill (1950)].

Clavimyrtus marginata Blume (1850) 115. - Jambosa marginata Miq. (1855) 428. - Type: Van Hasselt s.n. (holo L, not found).

Clavimyrtus virens Blume (1850) 114. - Jambosa virens Miq. (1855) 428. - Eugenia virens Koord. \& Valeton (1900) 113; Henderson (1949) 249, t. 47a. - Type: Van Hasselt s.n. (holo L, sh. 898.203-397), designated here. 
Eugenia fusiformis Duthie in Hook.f. (1878) 479. - Syzygium fusiforme Merr. \& Perry (1939) 176. - Type: Maingay 743 (holo K).

Eugenia leptogyna C.B. Rob. (1909) 368; Merr. (1923) 368; (1950) 392. - Type: FB 6839 (Merritt) (holo $\mathrm{PNH} \dagger$ ).

Distribution - Malesia: Malay Peninsula (Johor, Malaya, Perak (Singapore), Sumatra, Java, Borneo, Bali, and the Philippines (Luzon, Mindanao, Mindoro, Negros, Palawan, Panay).

Habitat - Forests, 0-1200 m altitude.

In view of the fact that the species was described locally several times it cannot be ruled out that the assertion by the Index Kewensis is correct and that the E Indian Eugenia ramosissima (Blume) Duthie belongs here as well:

Clavimyrtus ramosissima Blume (1850) 115. - Eugenia ramosissima Wall. [(1831) 3604, nom. nud.] ex Duthie in Hook.f. (1878) 480. - Jambosa ramosissima A.M. \& J.M. Cowan (1929) 67. - Syzygium ramosissimum N.P. Balakrishnan (1982, ‘1980’) 175 ('ramosissium’). - Type: Wallich Cat. 3604 (holo L, sh. 898.203-638), designated here.

Distribution - E India, Bangladesh.

\section{REFERENCES}

Amshoff, G.H.J. 1944. Myrtaceae. In: C.A. Backer, Noodflora voor Java IV-B, 1, Fam. 98: 22. Leiden.

Amshoff, G.H.J. 1945. Myrtaceae. In: C.A. Backer, Notes on the flora of Java II. Blumea 5: 500.

Backer, C.A. \& R.C. Bakhuizen van den Brink Jr. 1964. Flora of Java I: 343. Groningen.

Balakrishnan, N.P. 1982 ('1980') Nomenclatural notes on some flowering plants - III. Bull. Bot. Surv. India 22: 175.

Blume, C.L. 1826-1827. Bijdragen tot de flora van Nederlandsch-Indië 17: 1083. Batavia.

Blume, C.L. 1850. Myrtaceae, Subord. Myrteae. Mus. Bot. 1: 114-115.

Cowan, A.M. \& J.M. Cowan. 1929. The trees of northern Bengal: 67. Calcutta.

De Candolle, A.P. 1828. Prodromus systematis naturalis regni vegetabilis 3: 274, 287. Paris, etc.

Duthie, J.F. 1878. Myrtaceae. In: J.D. Hooker, Flora of British India 2: 479-480. London.

Henderson, M.R. 1949. The genus Eugenia (Myrtaceae) in Malaya. Gard. Bull. Singapore 12: 249, t. $47 \mathrm{a}$.

Koorders, S.H. \& T. Valeton. 1900. Bijdragen no. 6 tot de kennis der boomsoorten op Java. Meded. Lands Plantentuin 40: 110-113.

Korthals, P.W. 1847. Bijdragen tot de kennis der Myrtaceae. Ned. Kruidk. Arch. 1: 202.

Kuntze, O. 1891. Revisio generum plantarum 1: 239. Leipzig, etc.

Merrill, E.D. 1923. An enumeration of Philippine flowering plants 3: 368. Manila.

Merrill, E.D. 1950. Readjustments in the nomenclature of Philippine Eugenia species. Philipp. J. Sci. 79: 392-393.

Merrill, E.D. \& L.M. Perry. 1939. The myrtaceous genus Syzygium Gaertner in Borneo. Mem. Amer. Acad. Arts 18; Mem. Gray Herb. 4: 141, 176.

Miquel, F.A.W. 1855. Flora van Nederlandsch Indië 1, 1: 428. Amsterdam, etc.

Robinson, C.B. 1909. A preliminary revision of Philippine Myrtaceae. Philipp. J. Sci., Bot. 4: 368.

Swartz, O. 1788. Flora indiae occidentalis: 78. London.

Turner, I.M. 1996. What should the kelat trees of Malaya be called? J. Singapore Natl. Acad. Sci. 22-23: 15.

Turner, I.M. 1997 ('1995'). A catalogue of the vascular plants of Malaya. Gard. Bull. Singapore 47: 376 .

Wallich, N. 1831. A numerical list of dried specimens ...: no. 3604. London. 